\title{
A Data-Driven Stochastic Optimization Approach to the Seasonal Storage Energy Management
}

\section{Journal Article}

Author(s):

Darivianakis, Georgios; Eichler, Annika (1); Smith, Roy (D); Lygeros, John (1)

Publication date:

2017-10

Permanent link:

https://doi.org/10.3929/ethz-b-000231975

Rights / license:

In Copyright - Non-Commercial Use Permitted

Originally published in:

IEEE Control Systems Letters 1(2), https://doi.org/10.1109/LCSYS.2017.2714426 


\title{
A Data-driven Stochastic Optimization Approach to the Seasonal Storage Energy Management
}

\author{
Georgios Darivianakis, Annika Eichler, Roy S. Smith and John Lygeros
}

\begin{abstract}
Several studies in the literature have shown the potential energy savings emerging from the cooperative management of the aggregated building energy demands. Sophisticated predictive control schemes have recently been developed that achieve these gains by exploiting the energy generation, conversion and storage equipment shared by the building community. A common difficulty with all these methods is integrating knowledge about the long term evolution of the disturbances affecting the system dynamics (e.g. ambient temperature and solar radiation). In this context, the seasonal storage capabilities of the system are difficult to be optimally managed. This paper addresses this issue by exploiting available historical data to $(i)$ construct bounds that confine with high probability the optimal charging trajectory of the seasonal storage, and $(i i)$ generate a piece-wise affine approximation of the value function of the energy stored in the seasonal storage at each time step. Using these bounds and value functions, we formulate a multistage stochastic optimization problem to minimize the total energy consumption of the system. In a numerical study based on a realistic system configuration, the proposed method is shown to operate the system close to global optimality.
\end{abstract}

Index Terms-Stochastic optimal control; Smart cities/houses

\section{INTRODUCTION}

A ROUND $20-40 \%$ of the total energy consumption in Europe and the US is attributed to the building sector [1]. In this context, significant savings can be envisaged by the cooperative management of the aggregated building energy demands via an energy hub. The energy hub provides the interface between the building community and the power grid by utilizing shared energy generation, conversion and storage equipment [2]. In this context, seasonal storage devices, which are typically expensive to be bought and installed, can be integrated in the building community.

Several model predictive control schemes [3]-[5] have been proposed that exploit the diversity of the available energy sources and equipment under uncertain operation conditions. Typically, these methods are limited to addressing problems with a few days prediction horizon. This is due to $(i)$ the intensive computational power needed to solve optimization problems with multiple time stages, and (ii) the inaccurate long term forecast of weather processes and building energy demands. However, limiting the prediction horizon of the problem to only a few days can severely underutilize the

This research was partially funded by CTI within the SCCER FEEB\&D, the Swiss National Science Foundation under the project IMES and the ETH Zurich Foundation.

The authors are with the Automatic Control Laboratory, Department of Electrical Engineering and Information Technology, ETH Zurich, 8092 Zurich, Switzerland. \{gdarivia, eichlean, rsmith, lygeros\}@control.ee.ethz.ch

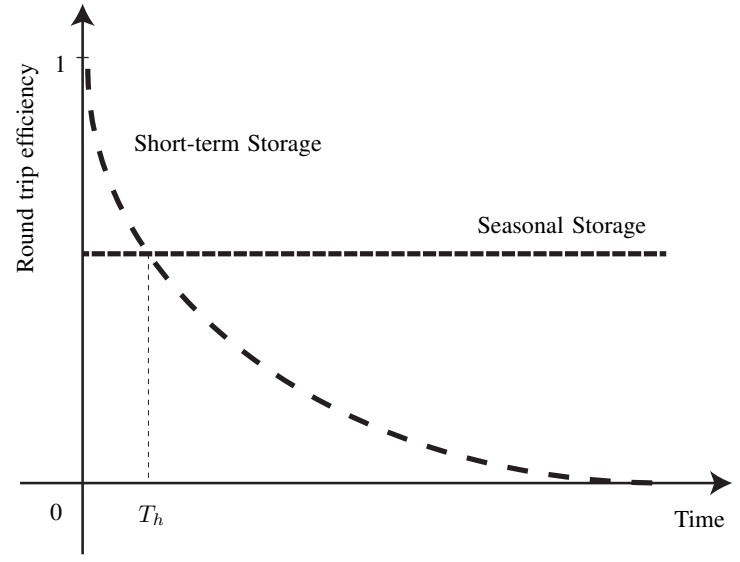

Fig. 1. Typical round trip efficiency curves of short-term and seasonal storage.

seasonal storage capabilities of the system. To illustrate the reason for this, in Fig. 1 the round-trip efficiency (RTE) of a seasonal and a short-term storage device are plotted versus time. It is apparent that seasonal storage is only utilized if the system decides to store energy for a period larger than $T_{h}$. In case of a battery as a short term storage with $0.3 \%$ decay rate and $90 \%$ charging/discharging efficiencies, and a typical seasonal storage (electrolyser, hydrogen tank, fuel cells) with $0 \%$ decay rate, $52 \%$ charging and $58 \%$ discharging efficiencies, we evaluated $T_{h} \simeq 13$ days. Hence, short prediction horizons make it hard the system to be operated in a cost efficient manner that can benefit from exploiting seasonalities appearing on photovoltaic energy production and electricity prices fluctuation.

There are several strategies in the literature that can deal with multistage stochastic optimization problems with long prediction horizons. The first one relies on the construction of a scenario tree to represent uncertainty and solve the problem as one large optimization problem [6]. This method strongly depends on the appropriate selection of a few representative scenarios of the evolution of the disturbances in the system. This however can be a hard task in case of limited knowledge about the distribution governing the evolution of the system disturbances. Other strategies, such as stochastic dual dynamic programminng (SDDP) [7]-[9] and approximate dynamic programming (ADP) [10], [11], are based on formulating the problem as a dynamic programming problem and then recursively constructing the value function at each time stage. In particular, the SDDP algorithm performs a sequence of major iterations known as the forward and the backward pass to build an approximately optimal value function, defined by 
cuts. On the other hand, ADP algorithms approximate the high-dimensional value function by a tractable approximation of much lower complexity that iteratively improves using simulated trajectories of the system evolution. The SDDP and ADP approaches are suitable for dealing with large scale systems for which their stochastic uncertainty is stage-wise independent or can be modelled by a Markov-process [11].

This paper proposes a data-driven stochastic predictive control scheme to operate, in a cost efficient manner, an energy hub with seasonal storage capabilities. The long term system operation is captured by a value function that rates the energy stored in the seasonal storage at the end of the horizon. Unlike SDDP and ADP approaches, the value function is evaluated based on available historical data of the system uncertainties, avoiding the necessity of modelling their underlying distributions. The novelty of the proposed method is that it exploits the available historical data to construct bounds that confine with high probability the optimal charging trajectory of the seasonal storage device. In the spirit of stochastic dynamic programming, the historical data are used to approximate the value function of the energy stored in the seasonal storage using piece-wise linear functions. Theoretical guarantees are provided on the worst-case error between the approximated and the real value function. Moreover, we formulate a multistage stochastic optimization problem to simultaneously minimize the total energy consumption of the system over a finite horizon and the value function of the seasonal storage at the end of the horizon. The bounds on the state of charge of the seasonal storage are imposed as a terminal constraints to the problem. We solve the resulting infinite dimensional optimization problem using a decision rule approximation. Our approach is data-driven due to the possibility of updating the bounds and value function approximation whenever new data becomes available.

The paper is organized as follows. In Section II, we briefly review the modelling approach and the stochastic optimization problem formulation. The main contributions are presented in Section III where we discuss the data-driven techniques associated with the derivation of the bounds on the state of charge of the seasonal storage, and the value functions approximation. Section IV provides a numerical study to assess the efficacy of the proposed method.

Notation: Random vectors are represented in boldface, while their realizations in normal font. Given vectors $v_{i} \in$ $\mathbb{R}^{k_{i}}$ with $k_{i} \in \mathbb{N}, i \in \mathcal{M}=\{1, \ldots, m\}$, we define $\left[v_{i}\right]_{i \in \mathcal{M}}=\left[v_{1}^{\top} \ldots v_{m}^{\top}\right]^{\top} \in \mathbb{R}^{k}$ with $k=\sum_{i=1}^{m} k_{i}$ as their vector concatenation. Given time dependent vectors $\nu_{t} \in \mathbb{R}^{\ell}$ with $\ell \in \mathbb{N}$, we define $\nu^{t}=\left[\nu_{1}^{\top} \ldots \nu_{t}^{\top}\right]^{\top} \in \mathbb{R}^{\ell t}$ as their history up to time $t$.

\section{PROBLEM DESCRIPTION}

The energy hub provides the interface between the building community and the energy grids by purchasing electricity and gas to serve the buildings energy demands for electricity and heating. As illustrated in the example Fig. 2, the energy hub operates a number of energy generation, conversion and storage devices (e.g., photovoltaics, battery, heat pump, chiller)

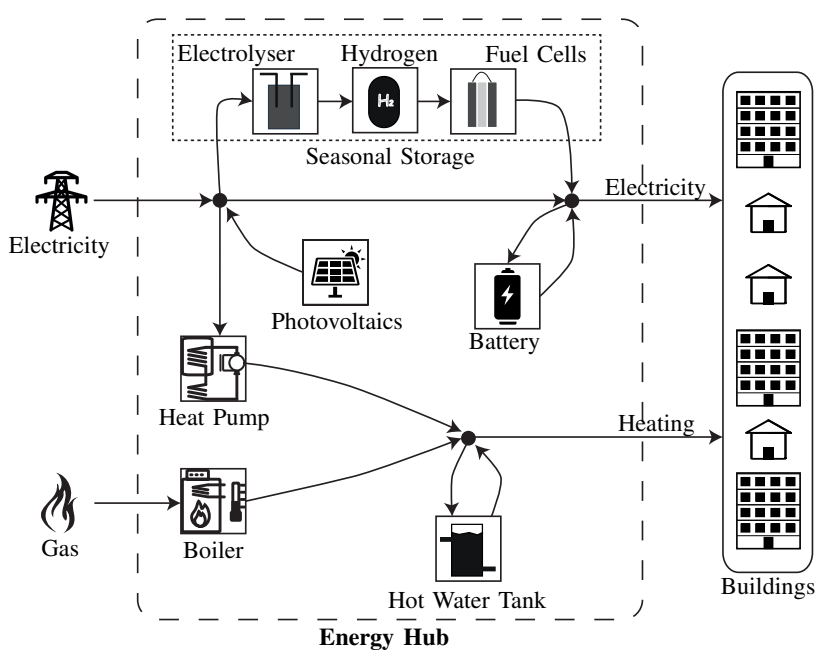

Fig. 2. Heating and electricity network of a building district.

that are shared by the building community. In this section, we describe the energy hub dynamics using discrete time, linear models affected by exogenous disturbances (e.g., solar radiation), and we discuss the formulation of the stochastic optimization problem associated with the optimal control of the energy hub devices.

\section{A. Dynamics, constraints and control laws}

We model the dynamics of the energy hub devices as,

$$
\boldsymbol{x}_{t+1}=A \boldsymbol{x}_{t}+B \boldsymbol{u}_{t}+C \boldsymbol{\xi}_{t},
$$

where the vectors $\boldsymbol{x}_{t}, \boldsymbol{u}_{t}$ and $\boldsymbol{\xi}_{t}$ concatenate all the states, inputs and disturbances affecting the energy hub devices at time $t \in \mathcal{T}$, where $\mathcal{T}$ is the set of time indexes over the prediction horizon $T$. The states considered here model the energy stored in the storage devices, since conversion and generation units are modelled as static maps. The matrices $A, B, C$ are assumed known and of appropriate dimensions.

We consider linear operational constraints for the energy hub devices (e.g., capacity limits), given as,

$$
E \boldsymbol{x}_{t}+F \boldsymbol{u}_{t}+G \boldsymbol{\xi}_{t} \leq h,
$$

with the matrices $E, F, G$ and $h$ considered known.

The electricity and heating energy carriers give rise to the following energy balancing constraint,

$$
H_{p} \boldsymbol{p}_{t}+H_{u} \boldsymbol{u}_{t}+H_{d} \boldsymbol{d}_{t}=\mathbf{0},
$$

where the matrices $H_{p}, H_{u}$ and $H_{d}$ are used to model the power flows affecting the respective balancing node. Here, $\boldsymbol{p}_{t}$ is the purchased power from the grid and $\boldsymbol{d}_{t}$ is the power demanded by the building community. In the following, we define the constraint set for the energy hub as,

$$
\mathrm{EH}_{t}\left(\boldsymbol{\xi}_{t}, \boldsymbol{d}_{t}\right)=\left\{\left(\boldsymbol{x}_{t}, \boldsymbol{u}_{t}, \boldsymbol{p}_{t}\right): \text { Equations (2) and (3) hold }\right\} \text {. }
$$

We assume that the disturbances $\boldsymbol{w}=\left[\boldsymbol{w}_{t}\right]_{t \in \mathcal{T}}$, where $\boldsymbol{w}_{t}=\left[\boldsymbol{\xi}_{t}, \boldsymbol{d}_{t}\right]$, is a random variable defined on a probability space $(\mathcal{W}, \mathcal{F}(\mathcal{W}), \mathbb{P}) . \mathcal{W}$ is a bounded metric space with the respective Borel $\sigma$-algebra $\mathcal{F}(\mathcal{W})$. We assume the 
existence of $N_{s}$ historical samples $\left(w^{1}, \ldots, w^{N_{s}}\right)$, which were independently drawn from $\mathbb{P}$. To obtain more samples, the available historical data can be exploited, as suggested in [5], to construct a set of distributions in which, with high probability, $\mathbb{P}$ is a member. Then, additional samples can be drawn from this distributional set. Notice that due to their dependence on $\boldsymbol{w}$, the variables $\left(\boldsymbol{x}_{t}, \boldsymbol{u}_{t}, \boldsymbol{p}_{t}\right)$ are also random.

\section{B. Stochastic model predictive control formulation}

The goal of the building community is to minimize the expected cost of purchased energy from the grid over a horizon while satisfying the constraints and dynamics of the devices in the system. At each time $t$, the energy hub makes its decisions based on the information $(i)$ on exogenous disturbances, $\boldsymbol{\xi}_{t}$, up to time $t-1$, and $(i i)$ on the realization of the energy demands, $\boldsymbol{d}_{t}$, up to $t$. Therefore, we model the decisions $\boldsymbol{p}_{t}, \boldsymbol{u}_{t} \in \mathcal{U}_{t}$ as being strictly causal with respect to system disturbances and causal with respect to the energy demands of the buildings. In this setting, $\boldsymbol{p}_{t}, \boldsymbol{u}_{t} \in \mathcal{U}_{t}$, where $\mathcal{U}_{t}$ is the infinite dimensional function space encoding this information, i.e., $\boldsymbol{p}_{t}\left(\boldsymbol{\xi}^{t-1}, \boldsymbol{d}^{t}\right)$ and $\boldsymbol{u}_{t}\left(\boldsymbol{\xi}^{t-1}, \boldsymbol{d}^{t}\right)$. The resulting stochastic optimization problem is given as,

$$
\begin{aligned}
& \min J(\boldsymbol{u}, \boldsymbol{p}) \\
& \left.\begin{array}{ll}
\text { s.t. } & \boldsymbol{p}_{t}, \boldsymbol{u}_{t} \in \mathcal{U}_{t}, \boldsymbol{w}_{t}=\left[\boldsymbol{\xi}_{t}, \boldsymbol{d}_{t}\right], \\
& \boldsymbol{x}_{t+1}=A \boldsymbol{x}_{t}+B \boldsymbol{u}_{t}+C \boldsymbol{\xi}_{t}, \\
& \left(\boldsymbol{x}_{t}, \boldsymbol{u}_{t}, \boldsymbol{p}_{t}\right) \in \mathrm{EH}_{t}\left(\boldsymbol{\xi}_{t}, \boldsymbol{d}_{t}\right),
\end{array}\right\} \forall t \in \mathcal{W},
\end{aligned}
$$

with decision variables $\left(\boldsymbol{p}_{t}, \boldsymbol{u}_{t}\right)$ for all $t \in \mathcal{T}$. The objective function $J(\cdot)$ is given as,

$$
J(\boldsymbol{u}, \boldsymbol{p})=\mathbb{E}_{\boldsymbol{w}}\left(\sum_{t \in \mathcal{T}} c_{t}^{\top} \boldsymbol{p}_{t}\right),
$$

where $c_{t}$ denotes the time varying costs of the input energy streams. Problem (4) is computationally intractable due to the infinite structure of its decision variables. However, as presented in [4], a computationally tractable finite dimensional approximation of this optimization problem is obtained by restricting its decision variables to admit an affine structure and then employing robust optimization techniques to reformulate it into a linear optimization problem [12].

Nevertheless, solving such an optimization problem within a reasonable amount of time can only be achieved for a limited prediction horizon. This is mainly due to the following two reasons: (i) Problem (4) scales polynomially with the number of decision stages, and $(i i)$ the prediction capabilities for the expected evolution of the uncertain variables, $\boldsymbol{d}_{t}$ and $\boldsymbol{\xi}_{t}$, are limited on a few days time ahead (e.g., COSMO-7 weather forecasts provided from the Swiss national weather service MeteoSwiss is limited to three days).

A commonly used approach [8], [9] to handle this issue is by extending the objective function in (5), as follows,

$$
J(\boldsymbol{u}, \boldsymbol{p})=\mathbb{E}_{\boldsymbol{w}}\left(\sum_{t \in \mathcal{T}} c_{t}^{\top} \boldsymbol{p}_{t}+V_{T+1}\left(\boldsymbol{x}_{T+1}\right)\right),
$$

where $V_{t}(\cdot)$ is usually referred as the value function; it actually gives a value to the energy stored in the storage devices of the system at time $t$.
Assumption 1: Over one year period, there exist at least one specific time, $t_{s}$, for which the stored energy in the system is zero.

This implies that $V_{t_{s}}\left(\boldsymbol{x}_{t_{s}}\right)=0$. Although restrictive, the above assumption typically holds for seasonal storage applications where the charging and discharging cycles mainly depend on the time of the year. For instance, the excess of energy produced by the photovoltaic units during summer is stored to be consumed during winter when demand is typically high; therefore, the seasonal storage should be empty sometime in the spring. Indeed, we verify the validity of this assumption in the numerical Section IV.

Due to Assumption 1 that enforces $V_{t_{s}}\left(\boldsymbol{x}_{t_{s}}\right)=0$, it is enough that the value function, $V_{t_{0}}\left(x_{t_{0}}\right)$, only captures the cost, in terms of purchased energy from the grid, of operating the system from time $t_{0}$ to $t_{s}-1$, if the energy initially stored in the storage devices is $x_{t_{0}}$. Note that $V_{t_{0}}\left(x_{t_{0}}\right)$ is the optimal value of Problem (4) with $\mathcal{T}=\left\{t_{0}, \ldots, t_{s}-1\right\}$.

Lemma 1: For each time $t$, the value function $V_{t}(\cdot)$ is monotonically decreasing in its arguments.

Proof: We prove this proposition by contradiction. To do so, we denote by $\left(p_{t_{0}}^{*}\left(x_{t_{0}}\right), u_{t_{0}}^{*}\left(x_{t_{0}}\right), x_{t_{0}}^{*}\left(x_{t_{0}}\right)\right)$ the optimizer of Problem (4) with $\mathcal{T}=\left\{t_{0}, \ldots, t_{s}-1\right\}$ and initial state of charge $x_{t_{0}}$. Assume now that given $x_{t_{0}}^{\prime}$ and $x_{t_{0}}^{\prime \prime}$ with $x_{t_{0}}^{\prime} \leq x_{t_{0}}^{\prime \prime}$, then the optimizers $\left(p_{t_{0}}^{*}\left(x_{t_{0}}^{\prime}\right), u_{t_{0}}^{*}\left(x_{t_{0}}^{\prime}\right), x_{t_{0}}^{*}\left(x_{t_{0}}^{\prime}\right)\right)$ and $\left(p_{t_{0}}^{*}\left(x_{t_{0}}^{\prime \prime}\right), u_{t_{0}}^{*}\left(x_{t_{0}}^{\prime \prime}\right), x_{t_{0}}^{*}\left(x_{t_{0}}^{\prime \prime}\right)\right)$ exist, and the respective optimal values satisfy $V_{t_{0}}\left(x_{t_{0}}^{\prime}\right) \leq V_{t_{0}}\left(x_{t_{0}}^{\prime \prime}\right)$. However, it is easy one to verify that $\left(p_{t_{0}}^{*}\left(x_{t_{0}}^{\prime}\right), u_{t_{0}}^{*}\left(x_{t_{0}}^{\prime}\right), x_{t_{0}}^{*}\left(x_{t_{0}}^{\prime}\right)\right)$ is also a feasible solution to every Problem (4) with initial state of charge $x_{t_{0}}^{\prime \prime} \geq x_{t_{0}}^{\prime}$ since the capacity limitations and operational constraints are satisfied. However, optimality implies that $V_{t_{0}}\left(x_{t_{0}}^{\prime}\right) \geq V_{t_{0}}\left(x_{t_{0}}^{\prime \prime}\right)$ which leads to a contradiction.

Evaluating the exact form of $V_{t}(\cdot)$ is a rather hard task with most studies in the literature developing techniques to approximate it [11].

\section{DATA-DRIVEN STOCHASTIC MODEL PREDICTIVE CONTROL FORMULATION}

In this paper, we restrict our attention to $V_{t}^{s}(\cdot)$ which is the value function associated with the seasonal storage. In this setting, the objective function in (6) is reformulated as,

$$
J^{s}(\boldsymbol{u}, \boldsymbol{p})=\mathbb{E}_{\boldsymbol{w}}\left(\sum_{t \in \mathcal{T}} c_{t}^{\top} \boldsymbol{p}_{t}+V_{T+1}^{s}\left(\boldsymbol{x}_{T+1}^{s}\right)\right),
$$

where we define $\boldsymbol{x}_{t}^{s}$ to be the subvector of $\boldsymbol{x}_{t}$ which denotes the states associated with the seasonal storage device. In the following, we develop a data-driven approach to construct a bounded set, $\mathcal{X}_{t}^{s}$, that confines the state of charge of the seasonal storage device. Additionally, the historical data are used to approximate $V_{t}^{s}\left(\boldsymbol{x}_{t}^{s}\right)$ for all $\boldsymbol{x}_{t}^{s} \in \mathcal{X}_{t}^{s}$. In this context, the variant of Problem (4) is given as follows,

$$
\begin{aligned}
\min & J^{s}(\boldsymbol{u}, \boldsymbol{p}) \\
\text { s.t. } & \boldsymbol{p}_{t}, \boldsymbol{u}_{t} \in \mathcal{U}_{t}, \boldsymbol{w}_{t}=\left[\boldsymbol{\xi}_{t}, \boldsymbol{d}_{t}\right], \\
& \boldsymbol{x}_{t+1}=A \boldsymbol{x}_{t}+B \boldsymbol{u}_{t}+C \boldsymbol{\xi}_{t}, \quad \forall \boldsymbol{w} \in \mathcal{W}, \\
& \left(\boldsymbol{x}_{t}, \boldsymbol{u}_{t}, \boldsymbol{p}_{t}\right) \in \mathrm{EH}_{t}\left(\boldsymbol{\xi}_{t}, \boldsymbol{d}_{t}\right), \forall \forall \in \mathcal{T} . \\
& \boldsymbol{x}_{T+1}^{s} \in \mathcal{X}_{T+1}^{s},
\end{aligned}
$$


Proposition 1: Problem (8) is a conservative approximation of Problem (4) formulated with objective (6) in the following sense: every feasible solution of Problem (8) is feasible in Problem (4), and the optimal value of Problem (8) is equal or larger than the optimal value of Problem (4).

Proof: The feasibility argument is proven considering that the constraint $\boldsymbol{x}_{T+1}^{s} \in \mathcal{X}_{T+1}^{s}$ restricts the feasible set of Problem (8) with respect to the one of Problem (4). To show the relation between the objective values of the two problems, we first define $\boldsymbol{x}_{t}^{n s}$ such that $\boldsymbol{x}_{t}=\left[\boldsymbol{x}_{t}^{s}, \boldsymbol{x}_{t}^{n s}\right]$ with $\boldsymbol{x}_{t}^{n s} \geq 0$ capturing the state of charge of the short-term storage devices. We now observe that $V\left(\boldsymbol{x}_{t}\right)=V\left(\boldsymbol{x}_{t}^{s}, \boldsymbol{x}_{t}^{n s}\right)$ and $V_{t}^{s}\left(\boldsymbol{x}_{t}^{s}\right)=V\left(\boldsymbol{x}_{t}^{s}, 0\right)$. By Lemma 1, we know that $V_{t}(\cdot)$ is monotonically decreasing on its arguments, therefore, $V_{t}^{s}\left(\boldsymbol{x}_{t}^{s}\right)=V_{t}\left(\boldsymbol{x}_{t}^{s}, 0\right) \geq V_{t}\left(\boldsymbol{x}_{t}^{s}, \boldsymbol{x}_{t}^{n s}\right)=V_{t}\left(\boldsymbol{x}_{t}\right)$.

In the rest of this paper, we denote by $\mathcal{T}_{y}$ the set including the time indexes, with hourly discretization, over a year time starting from $t_{s}$ and ending at $t_{s}-1$ (e.g., from 10 a.m. on April 1st 2016 to 9 a.m. on April 1st 2017).

\section{A. Seasonal storage bounds, $\mathcal{X}_{t}^{s}$, approximation}

The set $\mathcal{X}_{t}^{s}$ is defined as to confine with high probability the optimal trajectory for the state of charge of the seasonal storage device. To compute $\mathcal{X}_{t}^{s}$ we need to construct the optimal trajectory for the seasonal storage. This can be done by formulating Problem (4) over a yearly horizon $\mathcal{T}=\mathcal{T}_{y}$ for each historical sample $w^{i}$ with $i \in \mathcal{N}_{s}=\left\{1, \ldots, N_{s}\right\}$. The resulting deterministic optimization problem is given as,

$$
\left.\begin{array}{rl}
\min & J(u, p) \\
\text { s.t. } & p_{t}, u_{t} \in \mathcal{R}, w_{t}^{i}=\left[\xi_{t}^{i}, d_{t}^{i}\right], \\
& x_{t+1}=A \boldsymbol{x}_{t}+B \boldsymbol{u}_{t}+C \xi_{t}^{i}, \\
& \left(x_{t}, u_{t}, p_{t}\right) \in \operatorname{EH}_{t}\left(\xi_{t}^{i}, d_{t}^{i}\right),
\end{array}\right\} \forall t \in \mathcal{T}_{y} .
$$

where the decision variables $p_{t}, u_{t}$ now belong in the generic finite dimensional space $\mathcal{R}$. Notice that the objective functions $J(\cdot)$ given in (5) and (6) are identical due to the assumption that $V\left(x_{t_{s}}\right)=0$. In the following, we denote by $\left(\boldsymbol{p}^{*}\left(w^{i}\right), \boldsymbol{u}^{*}\left(w^{i}\right), \boldsymbol{x}^{*}\left(w^{i}\right)\right)$ the optimizer of Problem (9).

Assumption 2: Every Problem (9) is feasible and its feasibility domain has a nonempty interior. Moreover, the optimizer of Problem (9) exists and it is unique.

The existence and uniqueness of $\left(\boldsymbol{p}^{*}\left(w^{i}\right), \boldsymbol{u}^{*}\left(w^{i}\right)\right)$ are assumed here to streamline the presentation. Nevertheless, these assumptions can be removed since $(i)$ the existence of $\left(\boldsymbol{p}^{*}\left(w^{i}\right), \boldsymbol{u}^{*}\left(w^{i}\right)\right)$ mainly depends on the design of the system and should be taken as granted. To obtain a unique optimizer, one can typically use a tie-brake rule, e.g., selecting among the optimal solutions the one with the minimum Euclidean norm. The uniqueness of $\boldsymbol{x}^{*}\left(w^{i}\right)$ is also implied due to its explicit relation to $\boldsymbol{u}^{*}\left(w^{i}\right)$ through (1).

Proposition 2: The optimizer of Problem (9) generates a Borel measurable mapping from $(\mathcal{W}, \mathcal{F}(\mathcal{W}))$ to $(\mathcal{R}, \mathcal{F}(\mathcal{R}))$ that associates each $w^{i}$ with a unique $\left(\boldsymbol{p}^{*}\left(w^{i}\right), \boldsymbol{u}^{*}\left(w^{i}\right)\right)$.

Proof: The proof follows closely that of [13, Prop. 3.10] and is omitted in the interest of space.

We now approximate the sets $\mathcal{X}_{t}^{s}$ as a "box" given by $\left[\underline{x}_{t}^{s}, \bar{x}_{t}^{s}\right]$ with $\underline{x}_{t}^{s}$ and $\bar{x}_{t}^{s}$ denoting the lower and upper bounds, respectively. We define as $\underline{x}^{s}=\left[\underline{x}_{t}^{s}\right]_{t \in \mathcal{T}_{y}}, \bar{x}^{s}=\left[\bar{x}_{t}^{s}\right]_{t \in \mathcal{T}_{y}}$ the bounds over a year time, given as $\underline{x}^{s}=\underline{\alpha} \widetilde{x}^{s *}$ and $\bar{x}^{s}=\bar{\alpha} \widetilde{x}^{s *}$ where $\underline{\alpha}, \bar{\alpha}$ are scalar decision variables and $\widetilde{x}^{s *}$ is the trajectory derived as the mean value of the optimal trajectories $\boldsymbol{x}^{s *}\left(w^{i}\right)$ for $i \in \mathcal{N}_{s}$. The scaling factors $\underline{\alpha}$ and $\bar{\alpha}$ of the trajectory of the mean state of charge are computed as the solution of the following optimization problem,

$$
\min _{\underline{\alpha}, \bar{\alpha}}\left\{\|\underline{\alpha}-\bar{\alpha}\|: \underline{\alpha} \widetilde{x}^{s *} \leq \boldsymbol{x}^{s *}\left(w^{i}\right) \leq \bar{\alpha} \widetilde{x}^{s *}, \forall i \in \mathcal{N}_{s}\right\}
$$

The following theorem assess the violation probability $\epsilon$ by which the optimizer $\boldsymbol{x}^{s *}(\boldsymbol{w})$ will not lie between these bounds for any realization of the uncertain variable $\boldsymbol{w}$.

Theorem 1: Given the bounds $\underline{x}^{s}, \bar{x}^{s}$, and $\beta<1$, then

$$
\mathbb{P}\left[\boldsymbol{x}^{s *}(\boldsymbol{w}) \in\left[\underline{x}^{s}, \bar{x}^{s}\right]\right] \geq 1-\epsilon
$$

holds with confidence $1-\beta$, if $\epsilon$ is chosen as to satisfy,

$$
\epsilon \geq \frac{2}{N_{s}}\left(1+\ln \left(\frac{1}{\beta}\right)\right) .
$$

Proof: Following [14], we can derive the equivalent of Problem (10) chance-constrained problem, given as,

$$
\min _{\underline{\alpha}, \bar{\alpha}}\left\{\|\underline{\alpha}-\bar{\alpha}\|: \mathbb{P}\left[\boldsymbol{x}^{s *}(\boldsymbol{w}) \in\left[\underline{\alpha} \widetilde{x}^{s *}, \bar{\alpha} \widetilde{x}^{s *}\right]\right] \geq 1-\epsilon\right\} .
$$

It follows from [15] that the bounds derived in (10) are a feasible solution to Problem (13) with confidence $1-\beta$, if $\epsilon$ is chosen as the following inequality is satisfied,

$$
\sum_{j=0}^{\zeta-1}\left(\begin{array}{l}
N_{s} \\
j
\end{array}\right) \epsilon^{j}(1-\epsilon)^{N_{s}-j} \leq \beta .
$$

where $\zeta$ is the number of decision variables in Problem (10). An explicit upper bound on $\epsilon$ was established in [16], and is given by

$$
\epsilon \geq \frac{2}{N_{s}}\left(\zeta-1+\ln \left(\frac{1}{\beta}\right)\right) .
$$

In our case $\zeta=2$ since the only decision variables in Problem (10) are the scalars $\underline{\alpha}, \bar{\alpha}$.

\section{B. Value function approximation using discretization}

We have defined the value function, $V_{t}^{s}(\cdot)$, so as to capture the cost, in terms of purchased energy from the grid, of operating the system from time $t$ to $t_{s}$. To evaluate $V_{t_{0}}^{s}\left(x_{t_{0}}^{s}\right)$ for any $t_{0} \in \mathcal{T}_{y}$ and $x_{t_{0}}^{s} \in \mathcal{X}_{t_{0}}^{s}$, we need to compute the optimal value of Problem (4) formulated with $\mathcal{T}_{s}=\left\{t_{0}, \ldots, t_{s}-1\right\}$. Lacking any information regarding the support $\mathcal{W}$ and the expected evolution of $\boldsymbol{w}$, we are instead interested in evaluating the optimal value of the following randomized optimization problem,

$$
\left.\begin{array}{rl}
V_{t_{0}}^{s}\left(x_{t_{0}}^{s}\right)=\min & \frac{1}{N_{s}}\left(\sum_{t \in \mathcal{T}_{s}} c_{t}^{\top} p_{t}^{i}\right) \\
\text { s.t. } p_{t}^{i}, u_{t}^{i} \in \mathcal{R}, w_{t}^{i}=\left[\xi_{t}^{i}, d_{t}^{i}\right], \\
& x_{t+1}^{i}=A x_{t}^{i}+B u_{t}^{i}+C \xi_{t}^{i},, \forall i \in \mathcal{N}_{s}, \\
& \left(x_{t}^{i}, u_{t}^{i}, p_{t}^{i}\right) \in \mathrm{EH}_{t}\left(\xi_{t}^{i}, d_{t}^{i}\right),
\end{array}\right\} \forall t \in \mathcal{T}_{s} .
$$


We emphasize that the non-anticipativity assumption actually couples the decision variables $p_{t}^{i}, u_{t}^{i}$ of this problem, i.e., decision to be made at time $t$ depend only on information up to that time. As shown in [13, Thm 3.6], a probabilistic bound on the difference between the optimal values of Problem (4) and Problem (16) can also be provided. We now compute $V_{t}^{s}(\cdot)$ the optimal value of Problem (16) in $M$ ordered discrete points, $x_{t}^{s, m} \in \mathcal{X}_{t}^{s}$ with $m=1, \ldots, M$, and we then approximate $V_{t}^{s}(\cdot)$ using piecewise linear functions. In the following we denote this approximate by $\widetilde{V}_{t}^{s}(\cdot)$.

Theorem 2: The value function $\widetilde{V}_{t}^{s}(\cdot)$ is an upper approximation of $V_{t}^{s}(\cdot)$. For all $x_{t}^{s} \in\left[x_{t}^{s, m}, x_{t}^{s, m+1}\right]$, it holds,

$$
0 \leq \tilde{V}_{t}^{s}\left(x_{t}^{s}\right)-V_{t}^{s}\left(x_{t}^{s}\right) \leq L_{m}\left\|x_{t}^{s, m}-x_{t}^{s, m+1}\right\|,
$$

where $L_{m}$ is positive scalar.

Proof: Problem (16) is linear, therefore it is known that its value function $V_{t}^{s}\left(x_{t}^{s}\right)$ is convex and piece-wise affine [17]. Hence, there exist Lipschitz constant $L_{m}$ such that

$$
\left\|V_{t}^{s}\left(x_{t}^{s, m}\right)-V_{t}^{s}\left(x_{t}^{s, m+1}\right)\right\| \leq L_{m}\left\|x_{t}^{s, m}-x_{t}^{s, m+1}\right\|
$$

Due to the convexity and continuity of the value function, we also have that $\widetilde{V}_{t}^{s}\left(x_{t}^{s}\right) \geq V_{t}^{s}\left(x_{t}^{s}\right)$ for all $x_{t}^{s} \in \mathcal{X}_{t}^{s}$. To this end, for simplicity we assume the existence of a single state seasonal storage device, i.e., $x_{t}^{s} \in \mathbb{R}$. By Lemma 1, we know that $V_{t}^{s}(\cdot)$ is a monotonically decreasing; therefore, for all $x_{t}^{s} \in\left[x_{t}^{s, m}, x_{t}^{s, m+1}\right]$, it holds that

$$
\begin{aligned}
& \tilde{V}_{t}^{s}\left(x_{t}^{s}\right)-V_{t}^{s}\left(x_{t}^{s}\right) \\
& \leq \widetilde{V}_{t}^{s}\left(x_{t}^{s}\right)-V_{t}^{s}\left(x_{t}^{s, m+1}\right) \\
& =\alpha\left(x_{t}^{s}-x_{t}^{s, m}\right)+\widetilde{V}_{t}^{s}\left(x_{t}^{s, m}\right)-V_{t}^{s}\left(x_{t}^{s, m+1}\right) \\
& \leq \widetilde{V}_{t}^{s}\left(x_{t}^{s, m}\right)-V_{t}^{s}\left(x_{t}^{s, m+1}\right) \\
& =V_{t}^{s}\left(x_{t}^{s, m}\right)-V_{t}^{s}\left(x_{t}^{s, m+1}\right) \\
& \leq L_{m}\left\|x_{t}^{s, m}-x_{t}^{s, m+1}\right\|
\end{aligned}
$$

where $\alpha=\left(\widetilde{V}_{t}^{s}\left(x_{t}^{s, m+1}\right)-\widetilde{V}_{t}^{s}\left(x_{t}^{s, m}\right)\right) /\left(x_{t}^{s, m+1}-x_{t}^{s, m}\right) \leq 0$. The first implication follows from the monotonicity of $V_{t}^{s}(\cdot)$, the second from the linear approximation, while the last one from (18). The above inequality implies that the approximation error converges to zero as the dicretization becomes more dense. Extension to multiple seasonal storages is trivial by employing multidimensional planes instead of lines and is omitted in the interest of space.

We remark that another possibility for efficiently solving Problem (16) is to reformulate it as a dynamic program and perform a backward pass using piecewise affine approximations of the value function at each time step [10].

\section{NUMERICAL STUDY}

\section{A. Problem configuration}

We consider an energy hub similar to the one depicted in Fig. 2 that comprises several conversion (heat pump, boiler, electrolyser, fuel cell), storage (battery, hot water tank, hydrogen tank) and energy generation (photovoltaics) units. The energy hub is connected both to electricity and gas grid, and serves the demand for heating and electricity for the village of Zernez, consisting of approximately 300 buildings with various construction characteristics [18].
TABLE I

SUMMARY OF THE ENERGY HUB DEVICES.

\begin{tabular}{lrrr}
\hline Technology & Purchased Units & Capacity/Unit $(\mathrm{kW})$ & COP \\
\hline \hline Boiler & 45 & 100 & 0.8 \\
Heat pump & 194 & 14 & 3.2 \\
Fuel Cell & 2 & 160 & 0.58 \\
Electrolyser & 10 & 213 & 0.52 \\
Thermal Storage & 1 & 9798 & - \\
Hydrogen Storage & 1 & 3217542 & - \\
Battery & 1 & 394 & - \\
\hline
\end{tabular}

The sizing of the hub devices is summarized in Table I. The battery and the thermal storage admit a discharging rate of $0.3 \%$ and $1 \%$, respectively, while the hydrogen storage is assumed lossless. We consider a south oriented photovoltaic array with maximum output of $3.12 \mathrm{MW}$.

\section{B. Approximating $V_{t}^{s}(\cdot)$ and $\mathcal{X}_{t}^{s}$}

Using real weather data files from years 2008-2016, we formulated Problem (9) with a horizon of five years, and we identify $t_{s}$ as April 8th since around that time the seasonal storage device was always empty. Additionally, we randomly generated 100 weather scenarios, as described in [5], that serve as our extended historical data set. The energy demands for heating and electricity of the buildings with respect to each weather scenario are simulated using Energy Plus [19].

For each one of these disturbance scenarios, we solve Problem (9), and we depict in Fig. 3, the optimal trajectories for the state of charge of the battery and hydrogen storage. We observe that the optimal trajectories of the battery do not exhibit any similarities. This is due to the fact that the operation of temporary storage devices depends on the short term weather deviations which are different for each of the weather scenario considered. On the other hand, the state of charge of the seasonal storage device follows a very similar pattern for all the scenarios considered. This indicates that the optimal operation of the seasonal storage mainly depends on
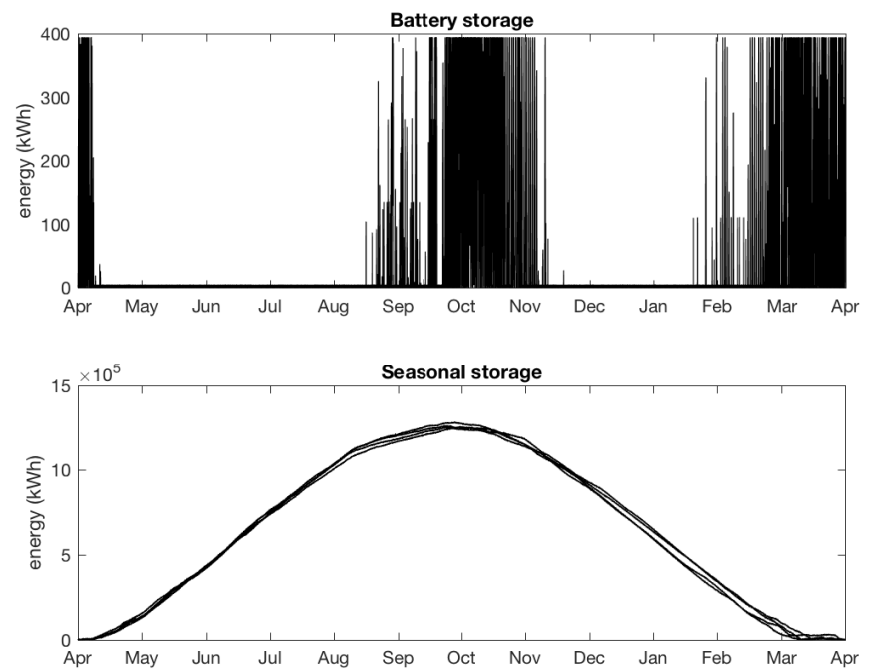

Fig. 3. Optimal state of charge trajectories of battery and seasonal storage devices for 108 randomly generated weather scenarios 
the seasonal characteristics of the weather disturbances, e.g., solar radiation is increased on summer days. $\mathcal{X}_{t}^{s}$ is obtained by lower and upper bounding these trajectories. Using Theorem 1, we calculated the probability that the optimal seasonal storage trajectory of the upcoming year lies outside these bounds as $\epsilon \simeq 0.08$ with confidence $\beta=0.05$.

We calculate $\widetilde{V}_{t}^{s}(\cdot)$ by solving Problem (16) only on the boundary points $\left(\underline{x}_{t}^{s}, \bar{x}_{t}^{s}\right)$. As expected, the obtained lines have a negative inclination that lies in the range $[-0.58,0]$.

\section{Performance evaluation}

We now investigate the importance of the value function $V_{t}^{s}(\cdot)$ and the bounds $\mathcal{X}_{t}^{s}$ on the performance of the system. We use as a metric the cost of purchased grid energy. To do so, we consider the following four variants of Problem (8):

- The No Terminal Conditions (NTC) formulation in which we disregard both $V_{t}^{s}(\cdot)$ and $\mathcal{X}_{t}^{s}$. This formulation is equivalent to solving Problem (4).

- The Terminal Constraint (TC) formulation in which we disregard $V_{t}^{s}(\cdot)$ while we keep $\mathcal{X}_{t}^{s}$.

- The Value Function (VL) formulation in which we keep $V_{t}^{s}(\cdot)$ while we disregard $\mathcal{X}_{t}^{s}$.

- The Terminal Constraint and Value Function (TCVL) formulation in which we keep both $V_{t}^{s}(\cdot)$ and $\mathcal{X}_{t}^{s}$.

We simulated the system over a year duration, in a recedinghorizon fashion, using a prediction horizon equal to 3 days and perfect forecast of the system disturbances. We compare the above mentioned cases to the optimal one in which we drop the receding horizon assumption and optimize over the whole year duration. The associated cost of each method for 25 randomly generated weather scenarios is reported in Table II with mean value and standard deviation. The mean values are normalized with respect to the mean of the optimal solution which is $441787 \mathrm{CHF}$.

TABLE II

PERFORMANCE COMPARISON OF DIFFERENT CONTROL STRATEGIES.

\begin{tabular}{c|cc} 
Method & Mean & Standard deviation \\
\hline \hline NTC & 1.134 & 0.0027 \\
TC & 1.078 & 0.0026 \\
VL & 1.060 & 0.0024 \\
TCVL & 1.002 & 0.0024 \\
Optimal & 1.000 & 0.0024 \\
\hline
\end{tabular}

We observe that the cost associated with the NTC strategy is significant since the system can not utilize its seasonal storage capabilities. On the other hand, the TC, VL and TCVL methods which include information about the optimal seasonal storage behavior in their problem formulation perform considerably better. Disregarding either $\mathcal{X}_{t}^{s}$ or $V_{t}^{s}(\cdot)$ from the problem formulation results in a slightly worse performance. An intuitive explanation for this is that the simple restriction of the seasonal storage charging state in some bounds does not provide any information about the value of the stored energy, while the approximation of the value function is poor outside the respective bounds. Instead, the proposed method exhibits improved performance that closely approximates the optimal one.

\section{CONCLUSION}

This paper presents a data-driven control framework for the problem of cooperatively managing aggregated building energy demands via an energy hub with seasonal storage capabilities. The proposed approach exploits available historical data to construct bounds that confine with high probability the optimal charging trajectory of the seasonal storage device. In addition, the value function of the seasonal storage is approximated using piece-wise affine functions. Theoretical guarantees are provided for the worst-case error of this value function approximation that depends on the discretization intervals. In a numerical study that is based on a realistic system set up, it is shown that the proposed approach achieves solutions that closely approximate the global optimal ones.

As future work, we intend to investigate the online adaptation of both the value function and the respective bounds, based on the real-time evolution of electricity and gas prices.

\section{REFERENCES}

[1] J. Laustsen. Energy efficiency requirements in building codes, energy efficiency policies for new buildings. Int. Energy Agency (IEA), pages 477-488, 2008

[2] M. Geidl, G. Koeppel, P. Favre-Perrod, B. Klock1, G. Andersson, and K. Frohlich. Energy hubs for the future. IEEE Power Energy Mag., 5(1):24-30, 2007

[3] A. Parisio, C. Del Vecchio, and A. Vaccaro. A robust optimization approach to energy hub management. Int. J. Elec. Power Energy Sys., 42(1):98-104, 2012.

[4] G. Darivianakis, A. Georghiou, R. S. Smith, and J. Lygeros. A stochastic optimization approach to cooperative building energy management via an energy hub. In Proc. IEEE Conf. Decision Control, pages 7814-7819, Osaka, Japan, 2015.

[5] G. Darivianakis, A. Georghiou, R. S. Smith, and J. Lygeros. The power of diversity: Data-driven robust predictive control for energy efficient buildings and districts. arXiv:1607.05441, 2016.

[6] S. Fleten and T. Kristoffersen. Short-term hydropower production planning by stochastic programming. Comput. Oper. Res., 35(8):26562671, 2008.

[7] M. Pereira and L. Pinto. Multi-stage stochastic optimization applied to energy planning. Math. Program., 52(1-3):359-375, 1991.

[8] G. Pritchard, A. Philpott, and P. Neame. Hydroelectric reservoir optimization in a pool market. Math. Program., 103(3):445-461, 2005.

[9] N. Löhndorf, D. Wozabal, and S. Minner. Optimizing trading decisions for hydro storage systems using approximate dual dynamic programming. Oper. Res., 61(4):810-823, 2013.

[10] D. Bertsekas. Dynamic programming and optimal control, volume 1. Athena Scientific Belmont, MA, 1995.

[11] W. Powell. Approximate Dynamic Programming: Solving the curses of dimensionality, volume 703. John Wiley \& Sons, 2007.

[12] A. Ben-Tal, A. Goryashko, E. Guslitzer, and A. Nemirovski. Adjustable robust solutions of uncertain linear programs. Math. Program., 99(2):351-376, 2004

[13] P. Esfahani, T. Sutter, and J. Lygeros. Performance bounds for the scenario approach and an extension to a class of non-convex programs. IEEE Trans. Autom. Control, 60(1):46-58, 2015.

[14] K. Margellos, P. Goulart, and J. Lygeros. On the road between robust optimization and the scenario approach for chance constrained optimization problems. IEEE Trans. Autom. Control, 59(8):2258-2263, 2014.

[15] M. Campi and S. Garatti. The exact feasibility of randomized solutions of uncertain convex programs. SIAM J. Optim., 19(3):1211-1230, 2008.

[16] G. Calafiore. Random convex programs. SIAM J. Optim., 20(6):34273464, 2010.

[17] T. Gal and J. Nedoma. Multiparametric linear programming. Manage. Sci., 18(7):406-422, 1972.

[18] K. Orehounig, G. Mavromatidis, R. Evins, V. Dorer, and J. Carmeliet. Towards an energy sustainable community: An energy system analysis for a village in Switzerland. Energy Buildings, 84:277-286, 2014.

[19] D. Crawley, L. Lawrie, C. Pedersen, and F. Winkelmann. Energy plus: Energy simulation program. ASHRAE J., 42(4):49-56, 2000. 